05

\title{
Низкочастотный спектр гиротропных мод конечной цепочки взаимодействующих ферромагнитных нанодисков
}

\author{
() В.А. Орлов, ${ }^{1,2}$ В.С. Прокопенко, ${ }^{3}$ Р.Ю. Руденко, ${ }^{1}$ И.Н. Орлова ${ }^{3}$ \\ ${ }^{1}$ Сибирский фредеральный университет, \\ 660041 Красноярск, Россия \\ ${ }^{2}$ Институт фоизики им. Л.В. Киренского ФИЦ КНЦ СО РАН, \\ 660036 Красноярск, Россия \\ ${ }^{3}$ Красноярский государственный педагогический университет им. В.П. Астафьева, \\ 660049 Красноярск, Россия \\ e-mail: orlhome@rambler.ru
}

Поступило в Редакцию 8 октября 2021 г.

В окончательной редакции 17 декабря 2021 г.

Принято к публикации 21 декабря 2021 г.

Рассмотрена задача о спектре частот коллективных гиротропных мод конечной линейной цепочки магнитостатически взаимодействующих ферромагнитных нанодисков. Показано, что информация о вихревом состоянии намагниченности каждого из нанодисков в цепочке может быть получена из анализа частотного спектра. Обсуждена перспектива использования регистрации спектра частот как способа считывания информации в устройствах магнитной памяти.

Ключивые слова: магнитный вихрь, ферромагнитный резонанс, коллективные моды.

DOI: 10.21883/JTF.2022.04.52243.273-21

\section{Введение}

Изучение магнитных наноструктур с вихревым распределением намагниченности остается актуальным и связано, в частности, с определенными ожиданиями и успехами в прикладном отношении (неинвазивная клеточная хирургия опухолей в медицине [1,2], устройства спинтроники [3-5]).

Вихревое состояние намагниченности $[6,7]$ характеризуется двумя параметрами: полярностью $p= \pm 1$ (направлением намагниченности в центре вихря, его „ядре“ по или против нормали к плоскости нанодиска) и киральностью $q= \pm 1$ (направлением циркуляции ориентации намагниченности в плоскости диска на его периферии - по или против часовой стрелки).

При определенных размерах и формах частиц вихревое распределение намагниченности является весьма устойчивым, что делает каждый такой объект отличным кандидатом для хранения бита информации. Шифровать состояние такого бита можно как полярностью, так и киральностью и их сочетаниями. Изменение состояния магнитной подсистемы таких дисков энергетически выгодно осуществлять приведя их в состояние резонансного возбуждения. При этом радиус траектории движения ядра вихря увеличивается, смещаясь к краю магнетика, и изменение полярности и/или киральности осуществляется относительно легко. Такое периодическое движение ядра называют гиротропным. Резонансные частоты гиротропного движения относительно невелики и составляют порядок сотен мегагерц. Методы управления магнитным состоянием объектов с вихревым распределением намагниченности разнооб- разны: с помощью коротких импульсов полей, ориентированных против полярности ядра вихря (см., например, [8]), с помощью полей кантелевера магнитосилового микроскопа [9], спин-поляризованными токами $[10,11]$.

Повышение плотности записи информации планируется достигать за счет уплотнения в расположении частиц на немагнитной матрице. Одновременно с этим остается целый ряд пока неразрешенных проблем. В частности, при плотном расположении частиц нельзя исключать их взаимное влияние, например, посредством магнитостатического взаимодействия. Взаимодействие приводит к возникновению коллективных мод, что влияет на спектр частот коллективных возбуждений намагниченности массива таких дисков [12-17] и затрудняет получение достоверной информации о магнитном состоянии каждого отдельного элемента.

Действительно, малый размер ферромагнитных объектов (зачастую десятки нанометров), в которых реализуется магнитный вихрь или скирмион, накладывает особые требования к инструментарию [18-21]. Но полученный в эксперименте спектр коллективных мод способен помочь восстановить информацию о магнитном состоянии отдельного элемента массива.

В большинстве теоретических работ решаются задачи по поиску спектров коллективных мод или больших массивов (где матрицу можно считать бесконечной), или цепочек из малого количества взаимодействующих элементов, поддающихся аналитическому счету. Но в больших массивах, как правило, по спектру невозможно восстановить информацию о магнитном состоянии отдельного элемента, а в цепочках из ма- 


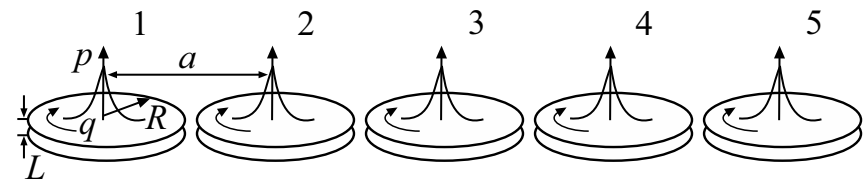

Рис. 1. Модель конечной цепочки ферромагнитных нанодисков.

лого числа элементов (например, 2 или 3) количество различимых магнитных состояний мало. Действительно, в паре частиц вне зависимости от того, какая их них является условно дефектной (первая или вторая с отличной, например, полярностью), наборы частот коллективных мод не будут различаться. В цепочке из трех частиц различимыми в эксперименте будут два состояния: дефектной является средняя частица или одна из крайних. По этой причине представляет слабый интерес и цепочка из четырех частиц. В цепочках из шести элементов(как будет видно ниже) наборы частот с дефектными частицами с номерами 3, 4 неразличимы. Это же касается массивов из большего числа элементов. Для семи частиц неразличимы состояния с выделенными частицами с номерами 3-5 и т.д. Т.е. увеличение числа элементов массива более пяти не представляется разумным, так как это не приводит к ожидаемо быстрому росту различимых по частоте состояний, которые позволили бы однозначно определить, какие из элементов являются дефектными.

По этой причине в настоящей работе мы теоретически рассматриваем компромиссный случай линейной цепочки из пяти элементов с вихревым распределением намагниченности. С одной стороны конечность цепочки позволяет надежно различить частоты нормальных мод коллективного движения ядер вихрей и по ним восстановить магнитные состояния элементов. С другой стороны, такая цепочка позволяет удовлетворить требованию миниатюризации устройства.

Задачей является получение спектра коллективных гиротропных мод цепочки из пяти магнитостатически взаимодействующих нанодисков с произвольным распределением полярностей и киральностей. Схематически цепочка изображена на рис. 1. Параметры нанодисков следующие: $R$ - радиус дисков, $L-$ толщина, $M_{S}-$ намагниченность насыщения ферромагнитного материала, $a$ - расстояние между центрами соседних дисков, элементы в цепочке имеют условную нумерацию от 1 до 5.

\section{1. Движения намагниченности в цепочке из одинаковых элементов}

Для описания движения намагниченности в объектах с вихревым распределением продуктивным является переход к коллективным переменным: координата и скорость ядра магнитного вихря. При этом эволюция намагниченности описывается подобно движению квази- частицы, в роли которой выступает ядро. Движение ядра описывается уравнением Тиля [22-24]:

$$
\mathbf{G} \times \mathbf{v}+\nabla W=0 .
$$

Здесь $\mathbf{G}=p C G_{0} \mathbf{k}-$ гировектор, направление которого перпендикулярно плоскости магнетика, $C$ - параметр, определяющий топологический заряд вихря (энергетически предпочтительным является состояние с $C=1[25]), \mathbf{k}-$ орт оси $z, G_{0}=2 \pi M_{S} L(1-p h) / \gamma$, $h=H / H_{S}$ - напряженность безразмерного магнитного поля, отнесенная к полю насыщения $H_{S}, \mathbf{H}=H \mathbf{k}$, $\mathbf{v}$ - скорость ядра магнитного вихря, $W$ - потенциальная энергия, которой обладает вихрь. В потенциальную энергию $W$ включаются слагаемые, ответственные за ее прирост вследствие выхода магнитной подсистемы элементов из метастабильного состояния (смещение ядра из равновесного положения), слагаемые, описывающие парную энергию взаимодействия между магнитными моментами разных элементов в цепочке и слагаемые, описывающие энергию вихря во внешних полях. В дальнейших расчетах мы пренебрежем затуханием, связанным с магнитными потерями.

Энергия ядра вихря представляется в виде

$$
W=\frac{\kappa \mathbf{r}^{2}}{2}+W_{\text {dip }}
$$

Здесь первое слагаемое ответственно за прирост энергии диска в результате смещения ядра из равновесного состояния (из центра диска), $\kappa=\frac{40}{9} \pi M_{S}^{2} L^{2}\left(1-h^{2}\right) / R-$ коэффициент квазижесткости магнитной подсистемы. Второе слагаемое описывает магнитостатическое взаимодействие магнитных моментов дисков.

Магнитные моменты $\mathbf{m}$ у нанодисков возникают при смещении ядра из центра. При этом взаимодействие между элементами массива можно интерпретировать как взаимодействие магнитостатических зарядов: объемных, имеющихся в толще магнетика, и поверхностных, возникающих на боковой поверхности дисков вследствие частичного выхода намагниченности за пределы магнетика [26,27]. В работе [26] авторы представили детальное теоретическое исследование механизмов магнитостатического взаимодействия элементов массивов. Было показано, что достаточно плотной упаковке дисков (расстояние между центрами менее удвоенного диаметра дисков [26]) дипольноедипольное приближение при оценке энергии связи требует уточнения и учета взаимодействия моментов высших порядков: дипольно-октупольного и октупольнооктупольного.

Часто эксплуатируется теоретическая модель так называемого жесткого вихря [28]. В рамках этой модели распределение намагниченности в теле магнитного вихря практически не меняется при смещении его ядра из центра диска. При этом конфигурация объемных магнитостатических зарядов неизменна, но на боковой 
поверхности возникает завышенная плотность поверхностных зарядов по сравнению с истинной [24,26]. Эта модель дает несколько завышенное значение энергии связи элементов, но позволяет относительно несложно провести теоретические оценки порядка величины расщепления частот.

Воспользуемся далее этой моделью, и предположим, что расстояние между центрами элементов достаточно велико, и диполь-дипольное приближение энергии взаимодействия не приводит к заметным ошибкам.

Так как магнитные моменты в теле вихря лежат в плоскости дисков (за исключением ядра), в дипольном приближении для магнитостатической энергии пары элементов можно записать

$$
W_{\mathrm{dip}}^{\prime}=\frac{\mathbf{m}_{1} \mathbf{m}_{2}}{a^{3}}-3 \frac{\left(\mathbf{m}_{1} \mathbf{a}\right)\left(\mathbf{m}_{2} \mathbf{a}\right)}{a^{5}} .
$$

Как показано в [29], направления моментов определяются ориентацией намагниченности на периметpe вихря $q= \pm 1$ и положением его ядра, которое в нашем случае удобно задавать в декартовых координатах $(x, y): m_{x}=-q m_{0} \sin (\phi)=-q m_{0} y / r$, $m_{y}=q m_{0} \cos (\phi)=q m_{0} x / r$. Здесь $m_{0}$ - модуль дипольного магнитного момента, который зависит от величины смещения ядра из центра $r$.

На рис. 2 показаны механизм возникновения зарядов на поверхности диска и схема, позволяющая оценить их величину и примерную величину дипольного момента. Поверхностная плотность заряда равна перпендикулярной к поверхности компоненте намагниченности: $\sigma=M_{S} \cos (\theta)=r \sin (\phi) / \sqrt{R^{2}+r^{2}-2 R r \cos (\phi)}$. Это выражение получено с использованием теорем

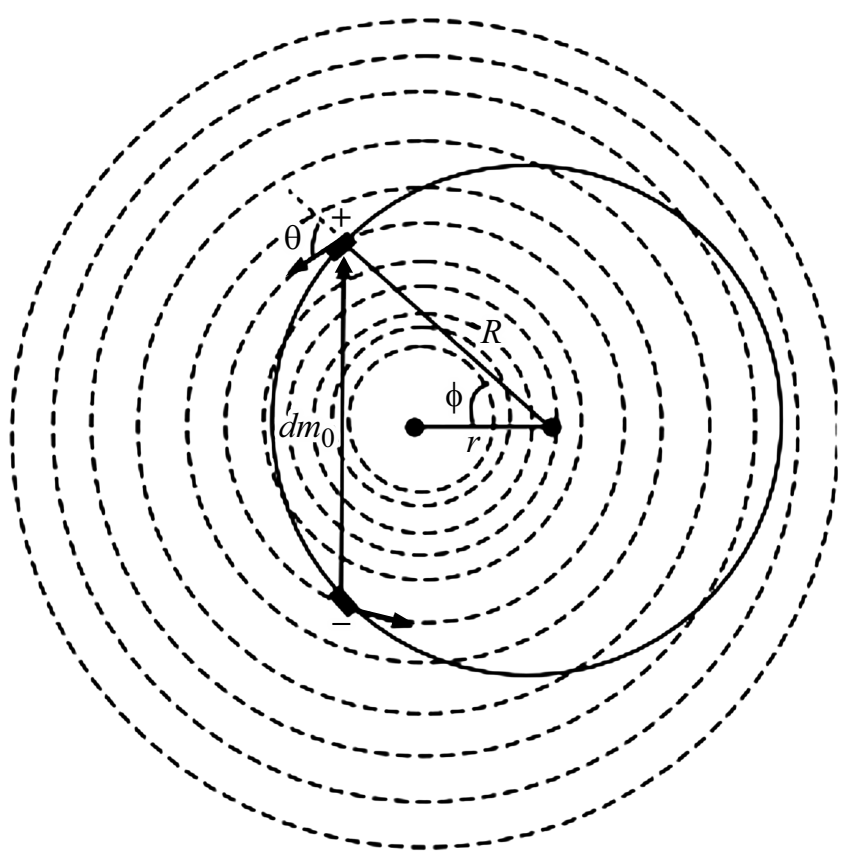

Pис. 2. Схема возникновения магнитного заряда и магнитного момента диска при смещении ядра вихря из центра частицы.

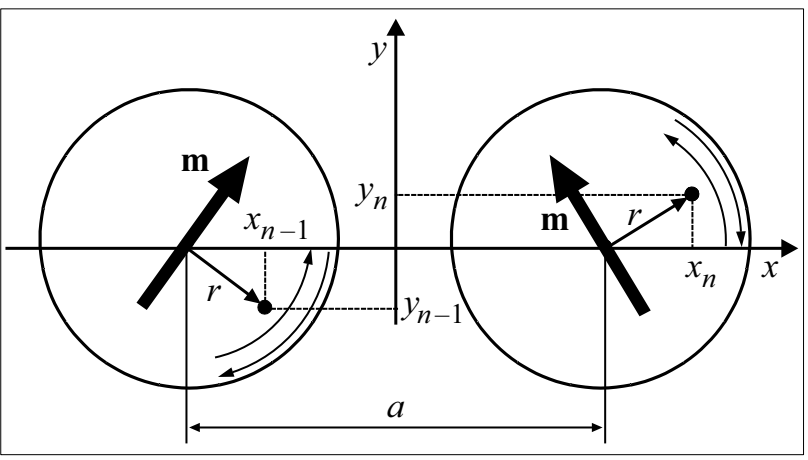

Рис. 3. Система координат и схема магнитостатического взаимодействия между дисками. Точкой показано положение ядра вихря, жирной стрелкой - направление магнитного момента. На обоих дисках выбрано направление намагниченности по часовой стрелке, тонкими стрелками у края дисков показаны возможные направления движения ядра.

синусов и косинусов применительно к треугольнику, построенному на сторонах $R$ и $r$ (рис. 2). Малый дипольный момент, образованный парой разноименных элементов поверхности, на рисунке обозначен как $d m_{0}$. Модуль полного магнитного момента можно оценить из выражения

$$
\begin{aligned}
m_{0}(r)= & \int_{0}^{\pi} 2 \sigma L R^{2} \sin (\phi) d \phi=2 M_{S} L R r \\
& \times \int_{0}^{\pi} \frac{\sin (\phi)^{2} d \phi}{\sqrt{1+\left(\frac{r}{R}\right)^{2}-2\left(\frac{r}{R}\right) \cos (\phi)}} .
\end{aligned}
$$

Численный расчет интеграла в выражении (4) показал, что он слабо меняется с увеличением отношения $r / R$, и лежит в диапазоне от $\pi / 2$ при $r / R=0$ до 4/3 при $r / R=1$. Для дальнейших оценок примем среднее значение интеграла $\approx 1.5$. Тогда для модуля магнитного момента можно приближенно записать: $m_{0} \approx 3 M_{S} L R r$.

В рассматриваемой здесь цепочке для расчета полной энергии магнитостатического взаимодействия необходимо вычислить сумму всех парных выражений вида (3) с учетом (4) (рис. 3):

$$
\begin{aligned}
W_{\mathrm{dip}} & =\sum W_{\mathrm{dip}}^{\prime}=\sum_{j \neq k}\left(\frac{\mathbf{m}_{j} \mathbf{m}_{k}}{a_{j k}^{3}}-3 \frac{\left(\mathbf{m}_{j} \mathbf{a}_{j k}\right)\left(\mathbf{m}_{k} \mathbf{a}_{j k}\right)}{a_{j k}^{5}}\right) \\
& =9 \frac{M_{S}^{2} L^{2} R^{2}}{a^{3}} \sum_{j \neq k} \frac{q_{j} q_{k}}{|k-j|^{3}}\left(x_{j} x_{k}-2 y_{j} y_{k}\right) .
\end{aligned}
$$

С учетом (2) и (5) уравнение (1) для, например, $j$-го элемента можно расписать по проекциям на декарто- 
вые оси:

$$
\left\{\begin{array}{l}
-v_{y_{j}}+\frac{\kappa_{j}}{G_{j}} x_{j}+9 \frac{M_{S}^{2} L^{2} R^{2} q_{j}}{a^{3} G_{j}} \sum_{k \neq j} \frac{q_{k} x_{k}}{|k-j|^{3}}=0, \\
v_{x_{j}}+\frac{\kappa_{j}}{G_{j}} y_{j}-18 \frac{M_{S}^{2} L^{2} R^{2} q_{j}}{a^{3} G_{j}} \sum_{k \neq j} \frac{q_{k} y_{k}}{|k-j|^{3}}=0 .
\end{array}\right.
$$

Далее возьмем производную по времени от обоих частей нижнего уравнения и подставим в результат величину $v_{y_{j}}$, выраженную из верхнего уравнения. В результате получим дифференциальное уравнение на только одну неизвестную компоненту:

$v_{x_{j}}^{\prime}+\frac{\kappa_{j}^{2}}{G_{j}^{2}} x_{j}+9 \frac{M_{S}^{2} L^{2} R^{2} q_{j}}{a^{3} G_{j}} \sum_{k \neq j} \frac{q_{k} x_{k}}{|k-j|^{3}}\left(\frac{\kappa_{j}}{G_{j}}-2 \frac{\kappa_{k}}{G_{k}}\right)=0$.

В этом уравнении отброшены слагаемые высшего порядка малости. Будем искать решение этого уравнения в виде $x_{j}=x_{0_{j}} e^{-i \omega t}$. После подстановки этого пробного решения в (7) получим систему алгебраических уравнений на комплексные амплитуды $x$-компоненты радиусвектора ядер дисков:

$$
\begin{aligned}
& \omega^{2} x_{0_{j}}=\omega_{0}^{2}\left(1+p_{j} h\right)^{2} x_{0_{j}} \\
& +\beta_{0}^{2} \sum_{k \neq j} \frac{x_{0_{k}} q_{j} q_{k}\left(1+p_{j} h-2 p_{j} p_{k}\left(1+p_{k} h\right)\right)}{|k-j|^{3}\left(1-p_{j} h\right)} .
\end{aligned}
$$

Здесь введены обозначения

$$
\omega_{0}^{2}=\left(\frac{20}{9} \frac{\gamma M_{S} L}{R}\right)^{2}, \quad \beta_{0}^{2}=10 \frac{\gamma^{2} M_{S}^{2} L^{2} R}{\pi a^{3}} .
$$

Если воспользоваться квантово-механическим формализмом, то систему уравнений вида (8) удобно представить в виде

$$
\widehat{\omega^{2}}\left|\mathbf{X}_{0}\right\rangle=\omega^{2}\left|\mathbf{X}_{0}\right\rangle
$$

Здесь $\left|\mathbf{X}_{0}\right\rangle$ - вектор, компонентами которого являются амплитуды $x_{0_{j}}, \widehat{\omega^{2}}$ - оператор „квадрата частоты“, который в полях, далеких от насыщения $(h \ll 1)$, для цепочки из пяти элементов с одинаковым магнитным состоянием имеет вид:

$$
\begin{aligned}
\widehat{\omega^{2}} & =(1+2 h) \\
& \times\left(\begin{array}{ccccc}
\omega_{0}^{2} & -\beta_{0}^{2} & -\frac{1}{8} \beta_{0}^{2} & -\frac{1}{27} \beta_{0}^{2} & -\frac{1}{64} \beta_{0}^{2} \\
-\beta_{0}^{2} & \omega_{0}^{2} & -\beta_{0}^{2} & -\frac{1}{8} \beta_{0}^{2} & -\frac{1}{27} \beta_{0}^{2} \\
-\frac{1}{8} \beta_{0}^{2} & -\beta_{0}^{2} & \omega_{0}^{2} & -\beta_{0}^{2} & -\frac{1}{8} \beta_{0}^{2} \\
-\frac{1}{27} \beta_{0}^{2} & -\frac{1}{8} \beta_{0}^{2} & -\beta_{0}^{2} & \omega_{0}^{2} & -\beta_{0}^{2} \\
-\frac{1}{64} \beta_{0}^{2} & -\frac{1}{27} \beta_{0}^{2} & -\frac{1}{8} \beta_{0}^{2} & -\beta_{0}^{2} & \omega_{0}^{2}
\end{array}\right) .
\end{aligned}
$$

Решить аналитически уравнение (10), т.е. собственные значения и вектора оператора (11), в общем случае не представляется возможным. Но численный расчет позволяет получить приближенные выражения для собственных значений оператора $\widehat{\omega^{2}}$.

Для соответствующих частот гиротропных мод будем иметь

$$
\omega^{2}=(1+2 h)\left(\begin{array}{c}
\omega_{0}^{2}-1.90 \beta_{0}^{2} \\
\omega_{0}^{2}-0.89 \beta_{0}^{2} \\
\omega_{0}^{2}+0.16 \beta_{0}^{2} \\
\omega_{0}^{2}+1.61 \beta_{0}^{2} \\
\omega_{0}^{2}+1.04 \beta_{0}^{2}
\end{array}\right)
$$

\section{2. Частоты мод цепочки с модулированным магнитным состоянием}

Рассмотрим далее практически интересные случаи, когда один или более элементов отличается своим состоянием от остальных элементов цепочки. Предположим, что полярность ядер магнитных вихрей всех элементов одинакова $\left(p_{j} p_{k}>0\right)$. Тогда из $(8)$ получим упрощенное выражение для элементов матрицы $\widehat{\omega^{2}}$ :

$$
\omega^{2} x_{0_{j}}=(1 \pm 2 h)\left(\omega_{0}^{2} x_{0_{j}}-\beta_{0}^{2} \sum_{k \neq j} \frac{x_{0_{k}} q_{j} q_{k}}{|k-j|^{3}}\right) .
$$

Таким образом, компоненты оператора определяются только сочетанием параметров $q$. Будем задавать состояние цепочки условным кодовым обозначением $\left\{q_{1} q_{2} q_{3} q_{4} q_{5}\right\}$. Для примера состояние $\{10000\}$ означает, что киральность только первого элемента цепочки отличается от остальных. Оператор для такого состояния имеет вид:

$$
\widehat{\omega^{2}}=(1 \pm 2 h)\left(\begin{array}{ccccc}
\omega_{0}^{2} & \beta_{0}^{2} & \frac{1}{8} \beta_{0}^{2} & \frac{1}{27} \beta_{0}^{2} & \frac{1}{64} \beta_{0}^{2} \\
\beta_{0}^{2} & \omega_{0}^{2} & -\beta_{0}^{2} & -\frac{1}{8} \beta_{0}^{2} & -\frac{1}{27} \beta_{0}^{2} \\
\frac{1}{8} \beta_{0}^{2} & -\beta_{0}^{2} & \omega_{0}^{2} & -\beta_{0}^{2} & -\frac{1}{8} \beta_{0}^{2} \\
\frac{1}{27} \beta_{0}^{2} & -\frac{1}{8} \beta_{0}^{2} & -\beta_{0}^{2} & \omega_{0}^{2} & -\beta_{0}^{2} \\
\frac{1}{64} \beta_{0}^{2} & -\frac{1}{27} \beta_{0}^{2} & -\frac{1}{8} \beta_{0}^{2} & -\beta_{0}^{2} & \omega_{0}^{2}
\end{array}\right) .
$$

Данная матрица отличается от (11) только знаками элементов строки и столбца, которым принадлежит дефектный элемент. Решение уравнения (10) для данного состояния не отличается от (12). Таким образом, спектр частот вырожден относительно изменения направления закручивания намагниченности в теле вихря.

Рассмотрим далее состояния цепочки, в которой для определенности параметры $q$ всех элементов одинаковы $\left(q_{j} q_{k}>0\right)$, а различаются направления намагниченности в ядрах вихрей - полярности $p$. Обозначения состояний остаются прежние: $\left\{p_{1} p_{2} p_{3} p_{4} p_{5}\right\}$. Выражение (8) при $h \ll 1$ принимает вид

$$
\begin{aligned}
\omega^{2} x_{0_{j}}= & \omega_{0}^{2}\left(1+2 p_{j} h\right) x_{0_{j}}+\beta_{0}^{2} \sum_{k \neq j} \frac{x_{0_{k}} q_{j} q_{k}}{|k-j|^{3}} \\
& \times\left(1-2 p_{j} p_{k}-2 p_{k} h\right) .
\end{aligned}
$$


Таблица 1. Значения параметра $\delta_{n}$ для массива элементов с одинаковой полярностью, но различающимися киральностями

\begin{tabular}{c|c|c|c|c|c}
\hline \multirow{2}{*}{ Киральность } & \multicolumn{5}{|c}{ Номер моды } \\
\cline { 2 - 6 } & 1 & 2 & 3 & 4 & 5 \\
\hline$\{01000\}$ & -4.47 & 4.20 & -1.11 & 0.16 & 1.22 \\
\hline$\{00100\}$ & -4.58 & 4.21 & -0.89 & 1.04 & 0.23 \\
\hline$\{11000\}$ & -3.60 & 3.14 & -0.81 & 0.21 & 1.06 \\
\hline$\{10100\}$ & -5.08 & 4.78 & -1.91 & 2.01 & 0.21 \\
\hline$\{10010\}$ & -4.66 & -2.81 & 0.22 & 3.05 & 4.20 \\
\hline$\{10001\}$ & -3.70 & -2.82 & 0.39 & 3.17 & 2.96 \\
\hline$\{01100\}$ & -4.07 & -2.31 & 3.22 & 2.92 & 0.24 \\
\hline$\{01010\}$ & -5.41 & -2.82 & 0.16 & 5.11 & 2.96
\end{tabular}

Таблица 2. Значения параметра $\xi_{n}$ для массива элементов с одинаковыми параметрами $q$, но различающимися полярностями $p$

\begin{tabular}{c|r|c|c|c|c}
\hline \multirow{2}{*}{ Полярность $p$} & \multicolumn{5}{|c}{ Номер моды $n$} \\
\cline { 2 - 6 } & 1 & 2 & 3 & 4 & 5 \\
\hline$\{01000\}$ & -0.62 & 0.01 & -2.11 & 0.32 & 2.39 \\
\hline$\{00100\}$ & -0.81 & 0.12 & -1.79 & 2.07 & 0.40 \\
\hline$\{11000\}$ & -0.28 & 0.06 & -1.24 & 0.30 & 1.80 \\
\hline$\{10100\}$ & -0.22 & 0.05 & -0.70 & 0.61 & 0.26 \\
\hline$\{10010\}$ & -0.77 & 0.13 & 0.40 & 0.24 & 0.00 \\
\hline$\{10001\}$ & -1.45 & 0.07 & 0.54 & 0.70 & 0.15 \\
\hline$\{01100\}$ & 0.67 & -1.14 & -0.87 & 0.96 & 0.38 \\
\hline$\{01010\}$ & -0.05 & -0.11 & 0.32 & -0.05 & -0.11
\end{tabular}

Таблица 3. Значения параметра $\varepsilon_{n}$ для массива элементов с одновременным изменением знака $p$ и $q$

\begin{tabular}{c|c|c|c|c|c}
\hline \multirow{2}{*}{ Параметры $p$ и $q$} & \multicolumn{5}{|c}{ Номер моды $n$} \\
\cline { 2 - 6 } & 1 & \multicolumn{1}{c|}{2} & 3 & 4 & 5 \\
\hline$\{01000\}$ & -4.47 & 4.20 & -1.11 & 0.16 & 1.22 \\
\hline$\{00100\}$ & -4.58 & 4.21 & -0.89 & 1.04 & 0.23 \\
\hline$\{11000\}$ & -3.60 & 3.14 & -0.81 & 0.21 & 1.06 \\
\hline$\{10100\}$ & -5.08 & 4.78 & -1.91 & 2.01 & 0.21 \\
\hline$\{10010\}$ & -4.66 & -2.81 & 0.22 & 3.05 & 4.20 \\
\hline$\{10001\}$ & -3.70 & -2.82 & 0.39 & 3.17 & 2.96 \\
\hline$\{01100\}$ & -4.07 & -2.31 & 3.22 & 2.92 & 0.24 \\
\hline$\{01010\}$ & -5.41 & -2.82 & 0.16 & 5.11 & 2.96
\end{tabular}

Таблица 4. Значения параметра $\chi_{n}$ для массива элементов с одновременным изменением знака $p$ и $q$

\begin{tabular}{c|r|c|r|c|c}
\hline \multirow{2}{*}{ Параметры $p$ и $q$} & \multicolumn{5}{|c}{ Номер моды $n$} \\
\cline { 2 - 6 } & 1 & 2 & \multicolumn{1}{c}{3} & 4 & 5 \\
\hline$\{10000\}$ & -1.21 & 0.49 & -2.28 & 0.40 & 2.59 \\
\hline$\{01000\}$ & -0.62 & 0.01 & -2.11 & 0.32 & 2.39 \\
\hline$\{00100\}$ & -0.81 & 0.12 & -1.79 & 2.07 & 0.40 \\
\hline$\{11000\}$ & -0.28 & 0.06 & -1.29 & -0.30 & 1.80 \\
\hline$\{10100\}$ & -0.22 & 0.05 & -0.70 & 0.61 & 0.26 \\
\hline$\{10010\}$ & -0.77 & 0.13 & 0.40 & 0.24 & 0.00 \\
\hline$\{10001\}$ & -1.49 & 0.11 & 0.54 & 0.73 & 0.11 \\
\hline$\{01100\}$ & 0.67 & -1.14 & -0.87 & 0.96 & 0.38 \\
\hline$\{01010\}$ & -0.05 & -0.11 & 0.32 & -0.05 & -0.11
\end{tabular}

В качестве примера приведем оператор, построенный по правилу (15) для состояния с распределением полярностей $\{10100\}$ :

$$
\widehat{\omega^{2}}=\left(\begin{array}{ccccc}
\omega_{0}^{2}(1-2 h) & \beta_{0}^{2}(3-2 h) & -\frac{1-2 h}{8} \beta_{0}^{2} & \frac{3-2 h}{27} \beta_{0}^{2} & \frac{3-2 h}{64} \beta_{0}^{2} \\
(3+2 h) \beta_{0}^{2} & \omega_{0}^{2}(1+2 h) & (3+2 h) \beta_{0}^{2} & -\frac{1+2 h}{8} \beta_{0}^{2} & -\frac{1+2 h}{27} \beta_{0}^{2} \\
-\frac{1-2 h}{8} \beta_{0}^{2} & (3-2 h) \beta_{0}^{2} & \omega_{0}^{2}(1-2 h) & (3-2 h) \beta_{0}^{2} & \frac{3-2 h}{8} \beta_{0}^{2} \\
\frac{3+2 h}{27} \beta_{0}^{2} & -\frac{1+2 h}{8} \beta_{0}^{2} & (3+2 h) \beta_{0}^{2} & \omega_{0}^{2}(1+2 h) & -(1+2 h) \beta_{0}^{2} \\
\frac{3+2 h}{64} \beta_{0}^{2} & -\frac{1+2 h}{27} \beta_{0}^{2} & \frac{3+2 h}{8} \beta_{0}^{2} & -(1+2 h) \beta_{0}^{2} & \omega_{0}^{2}(1+2 h)
\end{array}\right) .
$$

Результат расчета собственных значений операторов, построенных по правилу (15), удобно представить в виде

$$
\omega_{n}^{2}=\omega_{0}^{2}+\beta_{0}^{2} \delta_{n}+2 p_{n} \omega_{0}^{2} h+\beta_{0}^{2} \xi_{n} h .
$$

Значения параметров $\delta_{n}$ и $\xi_{n}$ приведены в табл. 1 и 2. Заметим, что нет необходимости приводить значения параметра $\xi$, например, для состояния $\{11011\}$, так как этот параметр для всех пяти собственных векторов будет отличаться лишь знаком от состояния $\{00100\}$. Очевидно, что для всех так называемых „негативных“ состояний физически поведение массива соответствует полю, включенному в обратном направлении. 
В качестве последнего примера рассмотрим вариант, когда выделенный диск в цепочке отличается от остальных знаками обеих параметров $\left(p_{j}=q_{j}= \pm 1\right)$. На основе правила $(15)$, как и ранее, можно строить матрицу оператора. В частном случае состояния $\{11000\}$ матрица имеет вид:

$$
\widehat{\omega^{2}}=\left(\begin{array}{ccccc}
\omega_{0}^{2}(1-2 h) & -\beta_{0}^{2}(1-2 h) & -\frac{3+2 h}{8} \beta_{0}^{2} & -\frac{3+2 h}{27} \beta_{0}^{2} & -\frac{3+2 h}{64} \beta_{0}^{2} \\
-(1-2 h) \beta_{0}^{2} & \omega_{0}^{2}(1-2 h) & -(3+2 h) \beta_{0}^{2} & -\frac{3+2 h}{8} \beta_{0}^{2} & -\frac{3+2 h}{27} \beta_{0}^{2} \\
-\frac{3-2 h}{8} \beta_{0}^{2} & -(3-2 h) \beta_{0}^{2} & \omega_{0}^{2}(1+2 h) & -(1+2 h) \beta_{0}^{2} & -\frac{1+2 h}{8} \beta_{0}^{2} \\
-\frac{3-2 h}{27} \beta_{0}^{2} & -\frac{3-2 h}{8} \beta_{0}^{2} & -(1+2 h) \beta_{0}^{2} & \omega_{0}^{2}(1+2 h) & -(1+2 h) \beta_{0}^{2} \\
-\frac{3-2 h}{64} \beta_{0}^{2} & -\frac{3-2 h}{27} \beta_{0}^{2} & -\frac{1+2 h}{8} \beta_{0}^{2} & -(1+2 h) \beta_{0}^{2} & \omega_{0}^{2}(1+2 h)
\end{array}\right) .
$$

Спектр частот мод в данном случае можно представить в виде

$$
\omega_{n}^{2}=\omega_{)}^{2}+\beta_{0}^{2} \varepsilon_{n}+2 p_{n} \omega_{0}^{2} h+\beta_{0}^{2} \chi_{n} h .
$$

Значения параметров $\varepsilon_{n}$ и $\chi$ приведены в табл. 3 и 4.

\section{Заключение}

Анализ таблиц поправок 1-4 показывает, что имеется потенциальная возможность различить состояния намагниченности цепочки с различными состояниями отдельных элементов. Для получения информации о состоянии конечной цепочки нет необходимости сканировать каждый элемент. Достаточно зафиксировать резонанс лишь части цепочки (например, только один выделенный элемент).

Для фиксации резонансных частот можно использовать оригинальную методику, предложенную в работе [30]. Авторы этой работы предложили способ надежной фиксации частот спектра с отдельного наноразмерного элемента с вихревой структурой с помощью магнито-резонасного силового микроскопа с доработанным кантилевером, на конце которого закреплен шарик из магнитомягкого материала. Величина взаимодействия шарика и магнитного момента ядра вихря достаточна для фиксации роста амплитуды движения кантилевера при увеличении радиуса движения ядра.

Следует заметить, что величина параметра $\beta_{0}$ может быть сравнима с собственной частотой движения вихря в изолированном диске $\omega_{0}$ даже в случае относительно слабого магнитостатического взаимодействия. Действительно, из выражений (9) следует отношение

$$
\psi=\frac{\beta_{0}}{\omega_{0}} \approx 0.8\left(\frac{R}{a}\right)^{\frac{3}{2}} .
$$

В случае, если расстояние между центрами дисков в два раза превышает их диаметр $(a=4 R)$, получим $\psi \approx 0.1$, что делает заметным слагаемые, пропорциональные $\beta_{0}^{2}$, и, следовательно, позволяет надежно различить частоты мод с различным магнитным состоянием цепочки. Продуктивным может быть метод, описанный в [30], или метод, представленный в [31], где авторы наблюдали резонанс в взаимодействующих дисках, фиксируя изменения сопротивления при пропускании переменного тока через отдельный элемент, или метод мягкой рентгеноскопии с временным разрешением [32].

Оценим абсолютные значения величин $\omega_{0}$ и $\beta_{0}$. Для наиболее часто используемого материала пермаллой с параметрами: $\frac{\gamma}{2 \pi}=2.8 \cdot 10^{6} \mathrm{MHz} / \mathrm{Oe}, M_{S}=750 \mathrm{Oe}$, $L / R=0.01, a / R=4$, расчеты с помощью выражений (9) дают $\frac{\omega_{0}}{2 \pi} \approx 65 \mathrm{MHz}, \frac{\beta_{0}}{2 \pi} \approx 6.5 \mathrm{MHz}$, что по порядку совпадает с результатами обстоятельного расчета для пары частиц, представленного в [26].

В случае присутствия магнитных перемычек между элементами (при непосредственном контакте между дисками) [33,34] магнитные подсистемы взаимодействуют не только магнитостатически, но не исключается и обменное взаимодействие. При этом рассуждения про зависимость частот мод от сочетания магнитных состояний элементов утрачивают силу, так как на фоне сильного обменного взаимодействия диполь-дипольное практически не заметно.

Важно отметить следующее обстоятельство. Резонасные частоты при модулировании магнитного состояния массива только изменением киральности не различаются - спектр вырожден. Увеличить надежность получения информации о типе модуляции (полярностью или сочетанием полярности и киральности) можно приложением постоянного перпендикулярного поля $h$. Смещение спектра резонансных частот существенно различно для этих случаев (см. (17) и (19)). Следовательно, для уверенного считывания состояния полярности необходимо включение перпендикулярного магнитного поля.

В итоге можем констатировать, что спектр резонансных частот цепочки, получаемый в эксперименте, позволяет с помощью табл. 1-4 получить информацию о распределении полярности ядер вихрей (за исключением вырожденных состояний, обсужденных выше). При необходимости это обстоятельство позволяет закодировать и считывать состояния цепочки из пяти элементов, не прибегая к исследованию магнитного состояния каждого отдельного элемента. Достаточно фиксировать сигнал на одном элементе.

Такой подход к исследованию магнитного состояния массива может быть предпочтительным, так как исключает необходимость наблюдения полярности/киральности вихря каждого отдельного наноэлемента, что позволит ускорить и упростить процедуру расшифровки магнитных состояний элементов конечных цепочек. 


\section{Финансирование работы}

Исследование выполнено при финансовой поддержке РФФИ в рамках научного проекта № 20-02-00696.

\section{Конфликт интересов}

Авторы заявляют, что у них нет конфликта интересов.

\section{Список литературы}

[1] T.N. Zamay, V.S. Prokopenko, S.S. Zamay, K.A. Lukyanenko, O.S. Kolovskaya, V.A. Orlov, G.S. Zamay, R.G. Galeev, A.A. Narodov, A.S. Kichkailo. Nanomaterials, 11, 1459 (2021). DOI: 10.3390/nano11061459

[2] M. Goiriena-Goikoetxea, D. Munoz, I. Orue, M.L. FernandezGubieda, J. Bokor, A. Muela, A. Garcia-Arribas. Appl. Phys. Rev., 7, 011306 (2020). DOI: 10.1063/1.5123716

[3] G.A. Meshkov, A.P. Pyatakov, A.D. Belanovsky, K.A. Zvezdin, A.S. Loginov. J. Magn. Soc. Jpn., 36, 46-48 (2012). DOI: $10.3379 / \mathrm{msjmag} .1108 \mathrm{M} 009$

[4] S. Luo, L. You. APL Mater., 9, 050901 (2021). DOI: $10.1063 / 5.0042917$

[5] Z.R. Yan, Y.Z. Liu, Y. Guang, K. Yue, J.F. Feng, R.K. Lake, G.Q. Yu, X.F. Han. Phys. Rev. Appl., 15, 064004 (2021). DOI: 10.1103/PhysRevApplied.15.064004

[6] E.R.P. Novais, P. Landeros, A.G.S. Barbosa, M.D. Martins, F. Garcia, A.P. Guimaraes. J. Appl. Phys., 110, 053917 (2011). DOI: $10.1063 / 1.3631081$

[7] K.Y. Guslienko. J. Magnetics, 24(4), 549-567 (2019). DOI: 10.4283/JMAG.2019.24.4.549

[8] B. Van Waeyenberge, A. Puzic, H. Stoll, K. W. Chou, T. Tyliszczak, R. Hertel, M. Faehnle, H. Brueckl, K. Rott, G. Reiss, I. Neudecker, D. Weiss, C.H. Back, G. Schuetz. Nature, 444, 461 (2006).

[9] V.L. Mironov, B.A. Gribkov, A.A. Fraerman, S.A. Gusev, S.N. Vdovichev, I.R. Karetnikova, I.M. Nefedov, I.A. Shereshevsky, J. Magn. Magn. Mater., 312, 153 (2007).

[10] K. Yamada, S. Kasai, Y. Nakatani, K. Kobayashi, H. Kohno, A. Thiaville, T. Ono. Nature Mater., 6, 269 (2007)

[11] S.-K. Kim, Y.-S. Choi, K.-S. Lee, K.Y. Guslienko, D.-E. Jeong. Appl. Phys. Lett., 91, 082506 (2007).

[12] Р.В. Горев, Е.В. Скороходов, В.Л. Миронов. ФТТ, 62 (9), 1349-1353 (2020). DOI: 10.21883/FTT.2020.09.49752.22H [R.V. Gorev, E.V. Skorokhodov, V.L. Mironov. Physics of the Solid State, 62 (9), 1513-1517 (2020). DOI: $10.1134 / \mathrm{S} 1063783420090085]$

[13] P.D. Kim, V.A. Orlov, R.Yu. Rudenko, A.V. Kobyakov, A.V. Lukyanenko, V.S. Prokopenko, I.N. Orlova, T.V. Rudenko. Eur. Phys. J. B, 91, 90 (2018). DOI: $10.1140 /$ epjb/e2018-90006-0

[14] R. Ishikawa, M. Goto, H. Nomura, Y. Suzuki. Appl. Phys. Lett., 119, 072402 (2021). DOI: 10.1063/5.0053797

[15] Е.Г. Екомасов, С.В. Степанов, К.А. Звездин, Н.Г. Пугач, Г.И. Антонов. ФММ, $122(3), \quad 212-220$ (2021). DOI: $\quad 10.31857 / \mathrm{S} 0015323021030050$ [E.G. Ekomasov, S.V. Stepanov, K.A. Zvezdin, N.G. Pugach, G.I. Antonov. Phys. Metals and Metallography, 122 (3), 197-204 (2021). DOI: $10.1134 / \mathrm{S} 0031918 X 21030054]$
[16] P.D. Kim, V.A. Orlov, R.Yu. Rudenko, V.S. Prokopenko, I.N. Orlova, A.V. Kobyakov. J. Magn. Magn. Mater. 440, 171-174 (2017). DOI: 10.1016/j.jmmm.2016.12.125

[17] A. Vogel, A. Drews, T. Kamionka, M. Bolte, G. Meier. PRL, 105, 037201 (2010). DOI: 10.1103/PhysRevLett.105.037201

[18] N. Locatelli, V.V. Naletov, J. Grollier, G. de Loubens, V. Cros, C. Deranlot, C. Ulysse, G. Faini, O. Klein, A. Fert. Appl. Phys. Lett., 98, 062501 (2011). DOI: 10.1063/1.3553771

[19] Iu.V. Vetrova, M. Zelent, J. Soltys, V.A. Gubanov, A.V. Sadovnikov, T. Scepka, J. Derer, R. Stoklas, V. Cambel, M. Mruczkiewicz. Appl. Phys. Lett., 118, 212409 (2021). DOI: $10.1063 / 5.0045835$

[20] M. Kuchibhotla, A. Talapatra, A. Haldar, A.O. Adeyeye. J. Appl. Phys., 130, 083906 (2021). DOI: 10.1063/5.0060689

[21] Chi-Ming Jin, Hai-Feng Du. Chin. Phys. B, 24, 128501 (2015). DOI: $10.1088 / 1674-1056 / 24 / 12 / 128501$

[22] A.A. Thiele. J. Appl. Phys., 45, 377 (1974). DOI: $10.1063 / 1.1662989$

[23] A.K. Zvezdin, K.A. Zvezdin. Bull. Lebedev Phys. Institute, 37 (8), (2010). DOI: 10.3103/S1068335610080038

[24] K.Yu. Guslienko, B.A. Ivanov, V. Novosad, Y. Otani, H. Shima, K. Fukamichi. J. Appl. Phys., 91, 8037 (2002). DOI: $10.1063 / 1.1450816$

[25] А.Ю. Галкин, Б.А. Иванов. ЖЭТФ, 1361(7), 87-104 (2009). J. Appl. Phys., 91, 8037 (2002). DOI: $10.1063 / 1.1450816$

[26] O.V. Sukhostavets, J. Gonzalez, K.Yu. Guslienko. Phys. Rev. B, 87, 094402 (2013). DOI: 10.1103/PhysRevB.87.094402

[27] O.V. Sukhostavets, K.Y. Guslienko. Appl. Phys. Express, 8, 023002 (2015). DOI: 10.7567/APEX.8.023002

[28] K.Yu. Guslienko. J. Nanoscience and Nanotechnology, 8, 2745-2760 (2008)

[29] П.Д. Ким, В.А. Орлов, Р.Ю. Руденко, В.С. Прокопенко, И.Н. Орлова, С.С. Замай. Письма в ЖЭТФ, $101(8)$, 620-626 (2015). DOI: 10.7868/S0370274X1508010X

[30] G. de Loubens, A. Riegler, B. Pigeau, F. Lochner, F. Boust, K.Y. Guslienko, H. Hurdequint, L.W. Molenkamp, G. Schmidt, A.N. Slavin, V.S. Tiberkevich, N. Vukadinovic, O. Klein. Phys. Rev. Lett., 102, 17602 (2009)

[31] S. Sugimoto, Y. Fukuma, S. Kasai, T. Kimura, A. Barman, Y. Otani. Phys. Rev. Lett., 106, 197203 (2011)

[32] H. Jung, K.-S. Lee, D.-E. Jeong, Y.-S. Choi, Y.-S. Yu, D.-S. Han, A. Vogel, L. Bocklage, G. Meier, M.-Y. Im, P. Fischer, S.K. Kim. Scientific Rep., 1(59) (2011). DOI: $10.1038 /$ srep00059

[33] J. Dou, S. C. Hernandez, C. Yu, M.J. Pechan, L. Folks, J.A. Katine, M.J. Carey. J. Appl. Phys., 107, 09B514 (2010). DOI: $10.1063 / 1.3358068 \mathrm{D}$

[34] Ю.П. Иванов, Е.В. Пустовалов, А.В. Огнев, Л.А. Чеботкевич. ФТТ, 51 (11), 2167-2170 (2009). [Yu.P. Ivanov, E.V. Pustovalov, A.V. Ognev, L A. Chebotkevich. Physics of the Solid State, 51 (11), 2300-2303 (2009). DOI: $10.1134 / \mathrm{S} 1063783409110171]$ 